\title{
CNO hydrogen burning studied deep underground
}

\author{
The LUNA Collaboration
}

D. Bemmerer ${ }^{1, a}$, F. Confortola ${ }^{2}$, A. Lemut ${ }^{2}$, R. Bonetti ${ }^{3}$, C. Broggini ${ }^{1}$, P. Corvisiero ${ }^{2}$, H. Costantini $^{2}$, J. Cruz $^{4}$, A. Formicola ${ }^{5}$, Zs. Fülöp ${ }^{6}$, G. Gervino ${ }^{7}$, A. Guglielmetti ${ }^{3}$, C. Gustavino ${ }^{5}$, Gy. Gyürky ${ }^{6}$, G. Imbriani ${ }^{8}$, A.P. Jesus ${ }^{4}$, M. Junker ${ }^{5}$, B. Limata ${ }^{8}$, R. Menegazzo ${ }^{1}$, P. Prati ${ }^{2}$, V. Roca ${ }^{8}$, D. Rogalla ${ }^{9}$, C. Rolfs ${ }^{10}$, M. Romano ${ }^{8}$, C. Rossi Alvarez ${ }^{1}$, F. Schümann ${ }^{10}$, E. Somorjai ${ }^{6}$, O. Straniero ${ }^{11}$, F. Strieder ${ }^{10}$, F. Terrasi ${ }^{9}$, and H.P. Trautvetter ${ }^{10}$

1 Istituto Nazionale di Fisica Nucleare (INFN), Sezione di Padova, via Marzolo 8, 35131 Padova, Italy

2 Dipartimento di Fisica, Università di Genova, and INFN, Genova, Italy

3 Istituto di Fisica, Università di Milano, and INFN, Milano, Italy

${ }^{4}$ Centro de Fisica Nuclear da Universidade de Lisboa, Lisboa, Portugal

${ }^{5}$ INFN, Laboratori Nazionali del Gran Sasso, Assergi, Italy

6 ATOMKI, Debrecen, Hungary

7 Dipartimento di Fisica Sperimentale, Università di Torino, and INFN, Torino, Italy

8 Dipartimento di Scienze Fisiche, Università di Napoli "Federico II", and INFN, Sezione di Napoli, Napoli, Italy

9 Seconda Università di Napoli, Caserta, and INFN, Sezione di Napoli, Napoli, Italy

10 Institut für Experimentalphysik III, Ruhr-Universität Bochum, Bochum, Germany

11 Osservatorio Astronomico di Collurania, Teramo, and INFN, Sezione di Napoli, Napoli, Italy

Received: 3 July 2005 /

Published online: 8 March 2006 - (C) Società Italiana di Fisica / Springer-Verlag 2006

\begin{abstract}
In stars, four hydrogen nuclei are converted into a helium nucleus in two competing nuclear fusion processes, namely the proton-proton chain (p-p chain) and the carbon-nitrogen-oxygen (CNO) cycle. For temperatures above 20 million kelvin, the CNO cycle dominates energy production, and its rate is determined by the slowest process, the ${ }^{14} \mathrm{~N}(\mathrm{p}, \gamma){ }^{15} \mathrm{O}$ radiative capture reaction. This reaction proceeds through direct and resonant capture into the ground state and several excited states in ${ }^{15} \mathrm{O}$. High energy data for capture into each of these states can be extrapolated to stellar energies using an R-matrix fit. The results from several recent extrapolation studies are discussed. A new experiment at the LUNA (Laboratory for Underground Nuclear Astrophysics) $400 \mathrm{kV}$ accelerator in Italy's Gran Sasso laboratory measures the total cross section of the ${ }^{14} \mathrm{~N}(\mathrm{p}, \gamma){ }^{15} \mathrm{O}$ reaction with a windowless gas target and a $4 \pi$ BGO summing detector, down to center of mass energies as low as $70 \mathrm{keV}$. After reviewing the characteristics of the LUNA facility, the main features of this experiment are discussed, as well as astrophysical scenarios where cross section data in the energy range covered have a direct impact, without any extrapolation.
\end{abstract}

PACS. 25.40.Lw Radiative capture - 26.20.+f Hydrostatic stellar nucleosynthesis $-29.17 .+\mathrm{w}$ Electrostatic, collective, and linear accelerators $-29.30 . \mathrm{Kv} \mathrm{X}$ - and $\gamma$-ray spectroscopy

\section{Introduction}

Stars generate energy and synthesize chemical elements in thermonuclear reactions [1]. Initially, hydrogen is burned to helium, and then, depending on the mass and chemical composition of the star, also heavier elements can be synthesized.

Hydrogen burning in stars can proceed through several different mechanisms, namely the proton-proton chain (p-p chain), several catalytic cycles called the CNO (carbon-nitrogen-oxygen) cycles [2] I, II, III, and the Hot-CNO cycle, the neon-sodium and the magnesium-

\footnotetext{
a e-mail: bemmerer@pd.infn.it
}

aluminium cycle [1]. The p-p I chain (in the following text simply called p-p chain) converts four protons into one ${ }^{4} \mathrm{He}$ nucleus; it is formed by the following nuclear reactions:

$$
{ }^{1} \mathrm{H}\left(\mathrm{p}, \mathrm{e}^{+} \nu\right)^{2} \mathrm{H}(\mathrm{p}, \gamma){ }^{3} \mathrm{He}\left({ }^{3} \mathrm{He}, 2 \mathrm{p}\right){ }^{4} \mathrm{He} .
$$

The p-p II and III chains also convert four protons into one ${ }^{4} \mathrm{He}$ nucleus, but are much less likely than the p-p I chain, at solar temperature [3] but also at higher temperatures.

The CNO cycles I and II are given by the following chains of reactions, respectively:

$$
\begin{aligned}
& { }^{12} \mathrm{C}(\mathrm{p}, \gamma){ }^{13} \mathrm{~N}\left(\beta^{+}\right){ }^{13} \mathrm{C}(\mathrm{p}, \gamma){ }^{14} \mathrm{~N}(\mathrm{p}, \gamma){ }^{15} \mathrm{O}\left(\beta^{+}\right){ }^{15} \mathrm{~N}(\mathrm{p}, \alpha){ }^{12} \mathrm{C}, \\
& { }^{15} \mathrm{~N}(\mathrm{p}, \gamma){ }^{16} \mathrm{O}(\mathrm{p}, \gamma){ }^{17} \mathrm{~F}\left(\beta^{+}\right){ }^{17} \mathrm{O}(\mathrm{p}, \alpha){ }^{14} \mathrm{~N}(\mathrm{p}, \gamma){ }^{15} \mathrm{O}\left(\beta^{+}\right){ }^{15} \mathrm{~N}
\end{aligned}
$$




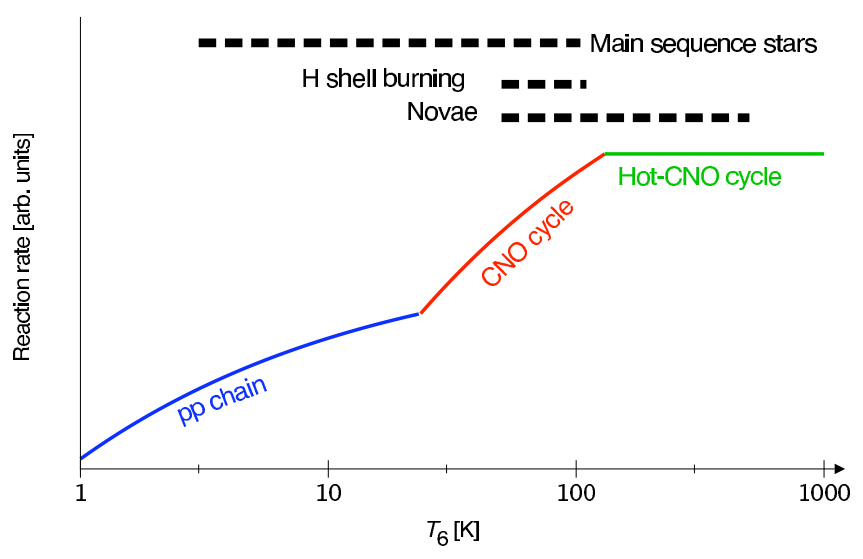

Fig. 1. The rate of energy generation of the three most important mechanisms of stellar hydrogen burning. The temperature of the transitions between the three regimes shown depends on the density and chemical composition of the star. Some relevant stellar burning scenarios [5] are indicated in the figure.

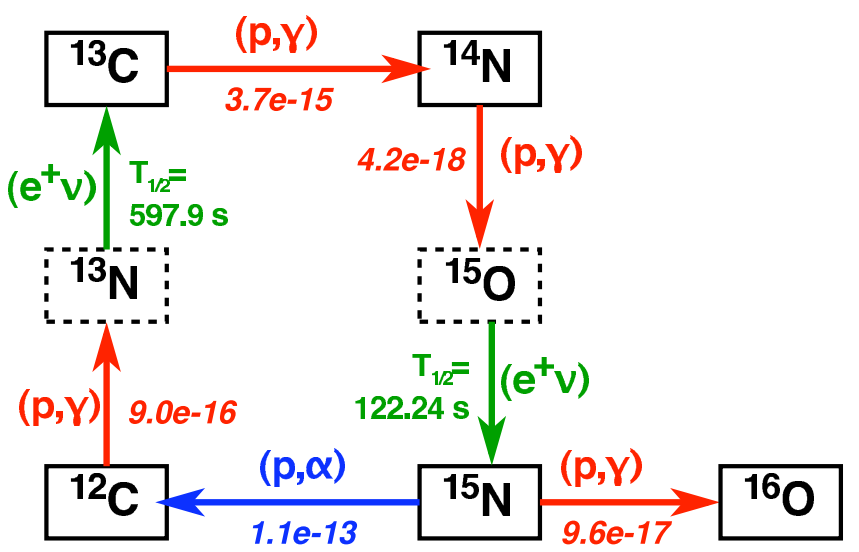

Fig. 2. The reactions of the CNO cycle [1]. Given are the NACRE [4] thermonuclear reaction rates $N_{\mathrm{A}}\langle\sigma v\rangle$ in $\frac{\mathrm{cm}^{3}}{\mathrm{~mol} \cdot \mathrm{s}}$ at the temperature at the center of our sun $\left(T_{6}=16\right)$.

These two cycles, as well as the less likely CNO cycles III and IV [1], also burn four protons into one ${ }^{4} \mathrm{He}$ nucleus. At higher stellar temperatures, the CNO cycles are supplanted by the so-called Hot-CNO cycles. The onset of the Hot-CNO cycles takes place when radiative capture on an unstable nuclide in the regular CNO cycles proceeds more rapidly than the $\beta^{+}$decay of the same nuclide.

To give some approximate numbers, at low temperatures, $T_{6}<20$ ( $T_{6}$ indicates the temperature of the burning site in the star in $\left.10^{6} \mathrm{~K}\right)$, energy production is dominated by the p-p chain (fig. 1). For $20<T_{6}<130$, the $\mathrm{CNO}$ cycle I (for simplicity just called the CNO cycle) dominates, for a chemical composition like that of our sun. At $T_{6} \approx 130$ (for a typical density of $100 \frac{\mathrm{g}}{\mathrm{cm}^{3}}$ ), the rate of radiative proton capture on the unstable nuclide ${ }^{13} \mathrm{~N}$ becomes faster than its $\beta^{+}$decay, and the $\beta$-limited Hot$\mathrm{CNO}$ cycle then dominates energy production.

Over the entire energy region where the CNO cycle dominates, the ${ }^{14} \mathrm{~N}(\mathrm{p}, \gamma){ }^{15} \mathrm{O}$ reaction is its bottleneck (fig. 2). Therefore, the rate of this particular nuclear reaction determines the rate of the entire cycle.

The present work first proposes a nuclear energy range of interest for understanding stellar CNO burning. Extrapolations by different authors giving the rate of the CNO cycle at stellar energies are then reviewed. The most important features of the Laboratory for Underground $\mathrm{Nu}-$ clear Astrophysics (LUNA) are given. A new experiment measuring the total cross section of the ${ }^{14} \mathrm{~N}(\mathrm{p}, \gamma){ }^{15} \mathrm{O}$ reaction at energies $E=70-230 \mathrm{keV}^{1}$ is presented. The astrophysical impact of directly measured cross sections at such low energies is discussed.

Details of the ${ }^{14} \mathrm{~N}(\mathrm{p}, \gamma){ }^{15} \mathrm{O}$ cross sections $[6,7]$ obtained in the experiment described here will be published separately.

\section{Which nuclear energy range is of astrophysical interest?}

The rate of energy production in thermonuclear burning is obtained from the energy produced per reaction and the number of reactions taking place per second, called the rate. This Maxwellian averaged thermonuclear reaction rate is called $\langle\sigma v\rangle$ and is obtained by folding the Maxwell-Boltzmann velocity distribution, calculated for the temperature of the star, with the energy-dependent nuclear reaction cross section. More precisely, $\langle\sigma v\rangle$ is given by the relation $[1]$

$$
\langle\sigma\rangle=\int_{0}^{\infty} \varphi(v) \cdot v \cdot \sigma(v) \mathrm{d} v,
$$

where $v$ is the relative velocity of the two reaction partners, $\varphi(v)$ the velocity distribution (given by the Maxwell-Boltzmann distribution) and $\sigma(v)$ the nuclear reaction cross section. In the following discussion, the center of mass energy $E$ will be used instead of the relative velocity $v$.

For energies $E$ far below the Coulomb energy, the cross section $\sigma(E)$ of a charged particle induced reaction drops steeply with decreasing energy due to the Coulomb barrier in the entrance channel:

$$
\sigma(E)=\frac{S(E)}{E} e^{-2 \pi \eta}
$$

where $S(E)$ is the astrophysical $S$-factor [1], and $\eta$ is the Sommerfeld parameter with $2 \pi \eta=31.29 Z_{1} Z_{2} \sqrt{\frac{\mu}{E}}$. Here $Z_{1}$ and $Z_{2}$ are the atomic numbers of projectile and target nucleus, respectively, $\mu$ is the reduced mass (in amu), and $E$ is the center of mass energy (in $\mathrm{keV}$ ).

The derivative $\frac{\mathrm{d}\langle\sigma v\rangle}{\mathrm{d} E}$ forms the so-called Gamow peak, and its maximum is found at the Gamow energy $E_{\mathrm{G}}$. Because of the energy dependence of the cross section, the

\footnotetext{
${ }^{1}$ In the present work, $E$ denotes the energy in the center of mass system, and $E_{\mathrm{p}}$ is the projectile energy in the laboratory system.
} 


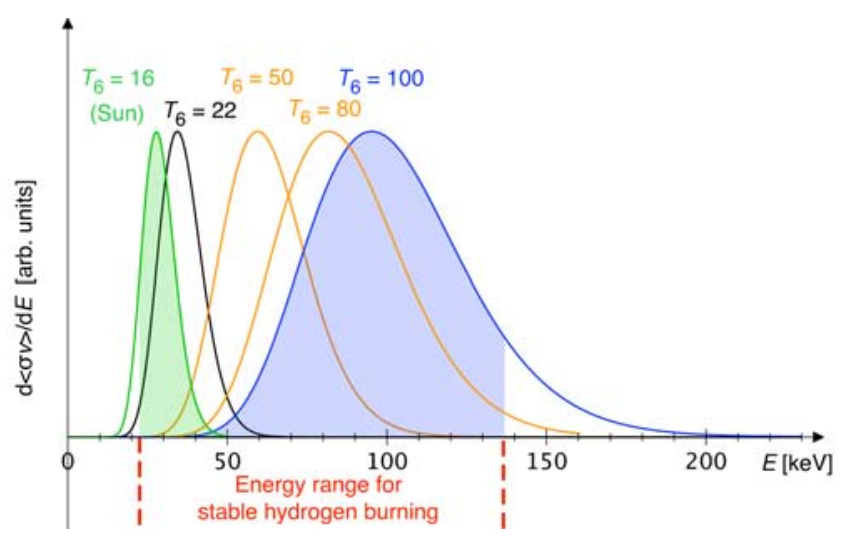

Fig. 3. Gamow peaks for the ${ }^{14} \mathrm{~N}(\mathrm{p}, \gamma){ }^{15} \mathrm{O}$ reaction in stable hydrogen burning scenarios. The peaks have been normalized to equal height. The shaded areas cover $90 \%$ of the integral under the respective Gamow peak.

Gamow energy is generally much higher than the temperature $k_{\mathrm{B}} T$ ( $k_{\mathrm{B}}$ : Boltzmann's constant) for the star. For example at solar temperature, $k_{\mathrm{B}} T=1.4 \mathrm{keV}$ and $E_{\mathrm{G}}=27 \mathrm{keV}$ for the ${ }^{14} \mathrm{~N}(\mathrm{p}, \gamma){ }^{15} \mathrm{O}$ reaction (fig. 3 ).

Hydrogen burning in stars on the main sequence of the Hertzsprung-Russell diagram [1] takes place at temperatures of the order of $T_{6}=3-100$, the latter value for very heavy primordial stars [8]. Temperatures of $T_{6}=50-80$ are typical for the hydrogen burning shell of an asymptotic giant branch (AGB) star of mass $M=2 M_{\odot}\left(M_{\odot}\right.$ : mass of our Sun) [9]. Higher temperatures are typical for explosive scenarios like novae [10] and X-ray bursts, which are not discussed here.

In the most recent solar model BS05 [11], the CNO cycle contributes only $0.8 \%$ of the solar luminosity, but a precise knowledge of its rate at $T_{6} \approx 16$, the temperature at the center of our Sun, can help test stellar evolution theory [3]. Low mass stars leave the main sequence in the Hertzsprung-Russell diagram towards the end of their life. The luminosity at this turnoff point depends on the CNO rate and can be used to determine the age of the star [12]; the larger the rate, the fainter the turnoff luminosity. This can be used to give an independent lower limit on the age of the universe $[13,14]$. The stellar temperature at this turnoff point is of the order of $T_{6} \approx 20$, depending on the star to be studied.

Using the temperatures indicated, one can propose an energy range of interest for understanding CNO hydrogen burning for the most important non-explosive stellar scenarios (fig. 3).

The cross section $\sigma(E)$ has a very low value at the resultant energies $E=20-140 \mathrm{keV}, \sigma(E)=10^{-22}-10^{-10}$ barn (eq. (2)). This prevents a direct cross section measurement in a laboratory at the earth's surface, where the signal to background ratio is too small because of cosmic ray interactions in detector, target, and shield. Hence, cross sections are measured at high energies and expressed as the astrophysical $S$-factor from eq. (2). The $S$-factor is then used to extrapolate the data to the relevant Gamow peak. Although $S(E)$ varies only slowly with energy for the direct nuclear reaction process, resonances and resonance tails may hinder an extrapolation, resulting in large uncertainties [1].

Therefore, the primary goal of experimental nuclear astrophysics remains to measure the cross section at energies inside the Gamow peak, or at least to approach it as closely as possible. The Laboratory for Underground Nuclear Astrophysics (LUNA) has been created for this purpose.

\section{The ${ }^{14} \mathrm{~N}(\mathrm{p}, \gamma){ }^{15} \mathrm{O}$ reaction}

\subsection{Situation up to the year 2000}

Up to the year 2000, there have been many experimental studies of the ${ }^{14} \mathrm{~N}(\mathrm{p}, \gamma){ }^{15} \mathrm{O}$ reaction at low energy (see, e.g., $[15,16,17,18,19,20])$. The energy levels in the ${ }^{15} \mathrm{O}$ nucleus are known ([21], fig. 4), and it is also known that only capture into the ground state and three excited states in ${ }^{15} \mathrm{O}$, at $5.181,6.172$, and $6.791 \mathrm{MeV}$, contributes significantly to the cross section at astrophysical energies [20].

Only one of the above named studies [16] obtained data that were at the edge of the astrophysically relevant energy region, with $50 \%$ statistical uncertainty for the cross section values. The other studies offer data only at energies above the astrophysical range, and generally, the

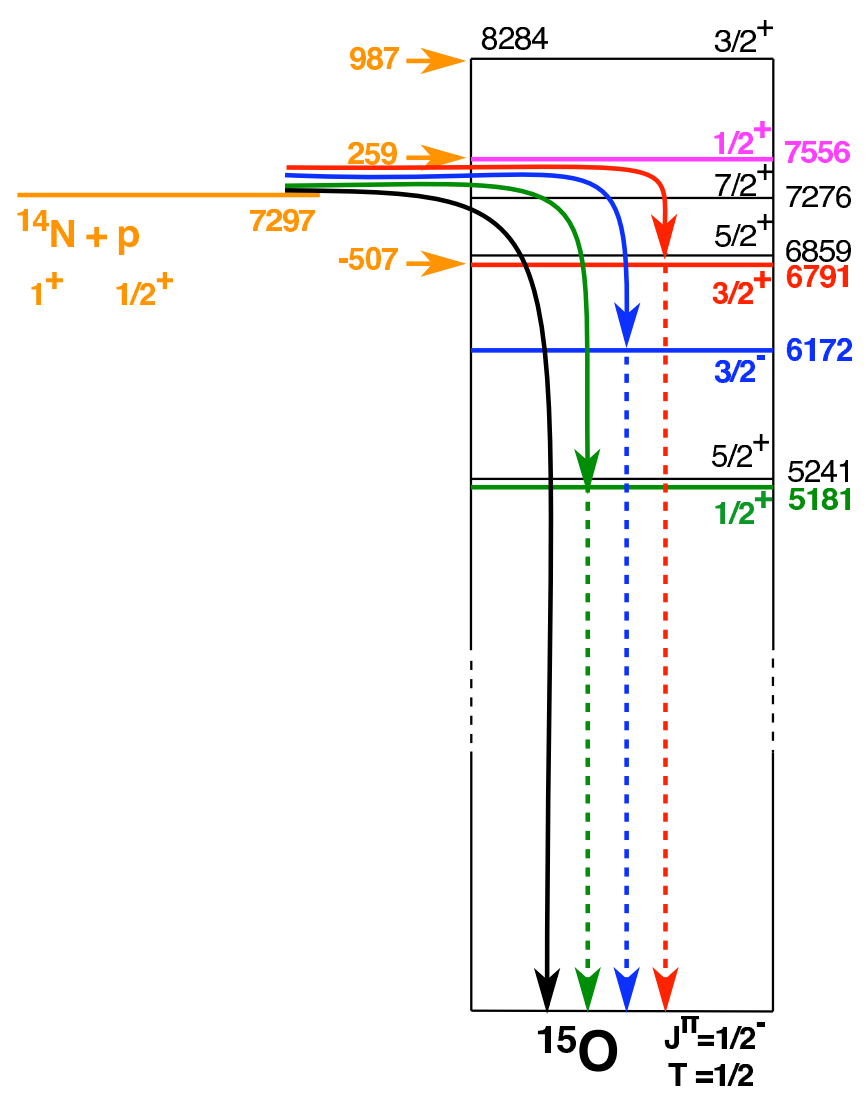

Fig. 4. Level scheme of ${ }^{15} \mathrm{O}$ up to $1 \mathrm{MeV}$ above the ${ }^{14} \mathrm{~N}+\mathrm{p}$ threshold according to [21]. For levels shown bold, the level energies are taken from the LUNA solid target experiment [22]. 
results are extrapolated in the framework of the R-matrix model down to stellar energies. The standard cross section value used in reaction rate compilations [23,4] is mainly based on the data of the comprehensive study by Schröder et al. [20] and on the low energy data from ref. [16].

After the year 2000, the R-matrix results of Schröder et al. [20] have been revised by several works, on theoretical [24], indirect $[25,26,27,28]$ and direct experimental [22, 29 ] grounds. The most dramatic revision was for capture to the ground state in ${ }^{15} \mathrm{O}$; the following section focuses on this particular transition.

\subsection{Recent R-matrix fits for radiative capture to the ground state in ${ }^{15} \mathrm{O}$}

The capture cross section into the ground state in ${ }^{15} \mathrm{O}$ is determined by destructive interference of direct capture amplitudes with resonant capture through the $6.79 \mathrm{MeV}$ state.

The direct capture can be parameterized with an asymptotic normalization coefficient (ANC) $C$ [30]. The total ANC for capture into the ground state $C=$ $\sqrt{C_{p_{1 / 2}}^{2}+C_{p_{3 / 2}}^{2}}$ (with two proton orbitals contributing) has been experimentally determined through the ${ }^{14} \mathrm{~N}\left({ }^{3} \mathrm{He}, \mathrm{d}\right){ }^{15} \mathrm{O}$ reaction in two independent recent studies from Triangle Universities (TUNL) in 2002 [26] and from Texas A\&M University (TAMU) in 2003 [27], with consistent results (table 1 ).

The most important parameter for resonant capture to the ground state via the $6.79 \mathrm{MeV}$ state (acting as a subthreshold resonance in this case) is the width $\Gamma_{\gamma, 6.79}$ of that state. This width has been measured with the Doppler shift attenuation method at TUNL in 2001 [25], and the obtained value could be confirmed in a Coulomb excitation study at RIKEN in 2004 [28] (table 2).

The LUNA 2004 study [22] measured the cross section for capture into the ground state in ${ }^{15} \mathrm{O}$ down to energies as low as $E=119 \mathrm{keV}$, much lower than any previous study for this transition, and directly confirmed the revised extrapolation for the ground state at those energies, inside the Gamow peak for some scenarios of stable hydrogen burning. Before, this revision had been based solely on theoretical and indirect considerations. In addition, the new low-energy data as well as previous data at higher energy, up to $2.5 \mathrm{MeV}$, from ref. $[20]^{2}$ were used for a new R-matrix fit (fig. 5).

The TUNL 2005 study [29] gave experimental data that are consistent with ref. [22], albeit with larger error bars. This study used its own experimental data $(E=$ $187-482 \mathrm{keV}$ for the ground state) also for an R-matrix fit (fig. 5), without including higher energy data in their fit. For comparison, also the 2003 R-matrix fit by the TAMU group [27] that is based on their ANC measurement and

\footnotetext{
2 In addition to presenting new, low energy data, the LUNA 2004 work [22] corrected the Schröder ground state data [20] for the summing-in effect and included this corrected data in the R-matrix fit.
}

Table 1. Asymptotic normalization coefficient $C$ for direct capture into the ground state in ${ }^{15} \mathrm{O}$ from different works.

\begin{tabular}{|l||c|c|l|}
\hline Group & $C\left[\mathrm{fm}^{-\frac{1}{2}}\right]$ & Method & Data from \\
\hline \hline Angulo 2001 [24] & 5.6 & fit & {$[20]$} \\
\hline TUNL 2002 [26] & $7.9 \pm 0.9$ & exp & {$[26,20]$} \\
\hline TAMU 2003 [27] & $7.3 \pm 0.4$ & exp & {$[27,20]$} \\
\hline LUNA 2004 [22] & 7.3 & fit & {$[22,20]$} \\
\hline TUNL 2005 [29] & $4.5-4.8$ & fit & {$[29]$} \\
\hline
\end{tabular}

Table 2. Gamma width of the state at $6.79 \mathrm{MeV}$ in ${ }^{15} \mathrm{O}$ from different works.

\begin{tabular}{|l||c|c|l|}
\hline Group & $\Gamma_{\gamma, 6.79}[\mathrm{eV}]$ & Method & Data from \\
\hline \hline Schröder 1987 [20] & $6.3 \pm 1.9$ & fit & {$[20]$} \\
\hline Angulo 2001 [24] & $1.75 \pm 0.60$ & fit & {$[20]$} \\
\hline TUNL 2001 [25] & $0.41_{-0.13}^{+0.34}$ & exp & {$[25]$} \\
\hline TAMU 2003 [27] & 0.35 & fit & {$[27,20]$} \\
\hline RIKEN 2004 [28] & $0.95_{-0.95}^{+0.60}$ & exp & {$[28]$} \\
\hline LUNA 2004 [22] & $0.8 \pm 0.4$ & fit & {$[22,20]$} \\
\hline TUNL 2005 [29] & $1.7-3.2$ & fit & {$[29]$} \\
\hline
\end{tabular}

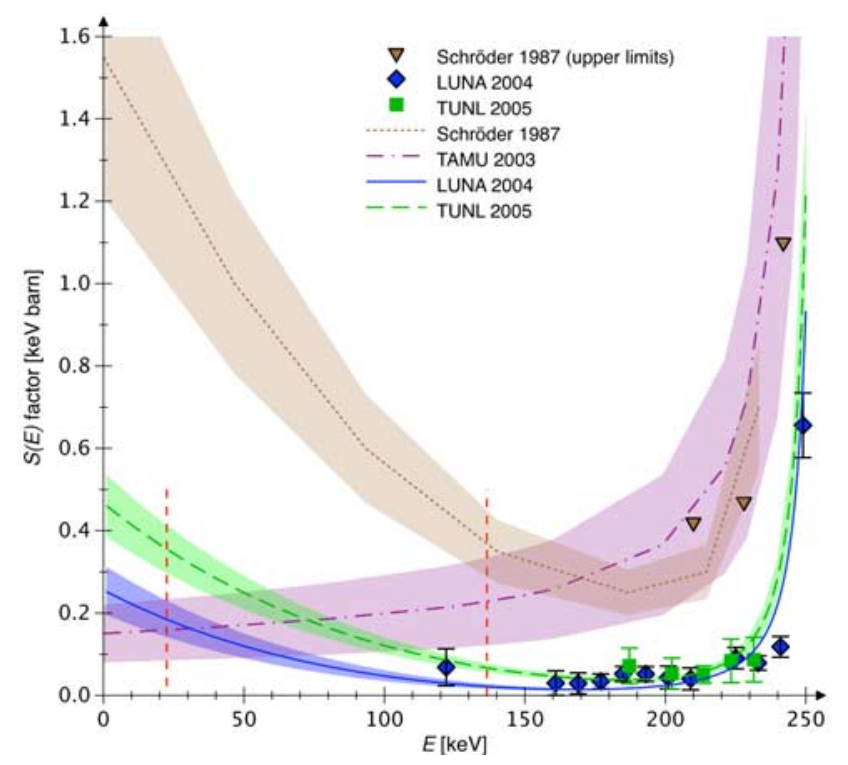

Fig. 5. Direct experimental data (inverted triangles: Schröder 1987 [20], upper limits; diamonds: LUNA 2004 [22]; squares: TUNL 2005 [29]) and R-matrix fits (lines) for capture to the ground state in ${ }^{15} \mathrm{O}$. The shaded areas around the lines correspond to the relative error for the extrapolated $S(0)$ value quoted by each of the studies. The vertical lines correspond to the energy range for stable hydrogen burning defined in fig. 3 .

normalized to the direct data from ref. [20] is included in the figure.

Figure 5 reveals interesting differences between the four extrapolations shown. The high $S(0)$ value from Schröder 1987 [20] is clearly dominated by the state at $6.79 \mathrm{MeV}$, here acting as a resonance $507 \mathrm{keV}$ below the reaction threshold. All other extrapolations shown use a much smaller $\Gamma_{\gamma, 6.79}$ value than Schröder 1987. Surprisingly, the fit by TUNL 2002 [26], not shown in the figure, 
Table 3. Extrapolated $S(0)$-factor for radiative proton capture into three states in ${ }^{15} \mathrm{O}$ from different works.

\begin{tabular}{|l||c|c|c|}
\hline $\begin{array}{l}\text { Capture into }{ }^{15} \mathrm{O} \\
\text { state with } E_{\mathrm{x}}=\end{array}$ & 6.791 & 6.172 & GS \\
\hline \hline Schröder 1987 $[20]$ & $1.41 \pm 0.02$ & $0.14 \pm 0.05$ & $1.55 \pm 0.34$ \\
\hline Angulo 2001 $[24]$ & $1.63 \pm 0.17$ & $0.06_{-0.02}^{+0.01}$ & $0.08_{-0.06}^{+0.13}$ \\
\hline TUNL 2002 [26] & $1.17 \pm 0.28$ & $0.14 \pm 0.03$ & $1.67 \pm 0.40$ \\
\hline TAMU 2003 [27] & $1.40 \pm 0.20$ & $0.13 \pm 0.02$ & $0.15 \pm 0.07$ \\
\hline Nelson 2003 [31] & 1.50 & $0.16 \pm 0.06$ & \\
\hline LUNA 2004 [22] & $1.35 \pm 0.05$ & & $0.25 \pm 0.06$ \\
\hline TUNL 2005 $[29]$ & $1.15 \pm 0.05$ & $0.04 \pm 0.01$ & $0.49 \pm 0.08$ \\
\hline
\end{tabular}

yields a similar rise of the $S$-factor to low energies, up to $S(0)=1.67 \mathrm{keV}$ barn, even though that study took solely direct capture into account.

The main difference between the input parameters used by the LUNA 2004 [22] and the TUNL 2005 [29] studies is that LUNA obtained a $\Gamma_{\gamma, 6.79}$ value that is much lower than the TUNL number (table 2). Both studies had left $\Gamma_{\gamma, 6.79}$ as a free parameter to fit their experimental excitation functions. An analogous approach was used by the same two studies regarding the ANC of the ground state, where LUNA obtains a $50 \%$ higher value than TUNL. The 2003 R-matrix fit by the TAMU group [27] used a value for $\Gamma_{\gamma, 6.79}$ that was very close to experiment, and the ANC used for the fit was obtained experimentally in the same work.

In summary, the results of different extrapolations (table 3) differ by more than the standard deviations quoted in the individual works, especially for capture into the ground state in ${ }^{15} \mathrm{O}$, but also for capture into the other two states contributing significantly, those at 6.172 and $6.791 \mathrm{MeV}$. It is therefore worthwhile to attempt a direct measurement of the cross section at energies of astrophysical interest.

\section{Laboratory for Underground Nuclear Astrophysics (LUNA)}

The Laboratory for Underground Nuclear Astrophysics (LUNA) has been designed for cross section measurements at energies in or near the Gamow peak. It is located in the Gran Sasso underground laboratory (Laboratori Nazionali del Gran Sasso, LNGS ${ }^{3}$ ) in Italy. LUNA uses high current accelerators with small energy spread in combination with high efficiency detection systems, one of which is described below.

The Gran Sasso facility consists of three experimental halls and several connecting tunnels. Its site is protected from cosmic rays by a rock cover equivalent to $3800 \mathrm{~m}$ water. This shield suppresses the flux of cosmic ray induced muons by six orders of magnitude [32], resulting in a flux of muon induced neutrons of the order of $\Phi_{\mathrm{n}_{\mu}} \approx 10^{-8} \frac{\mathrm{n}}{\mathrm{cm}^{2} \cdot \mathrm{s}}$ [33]. Because of neutrons from $(\alpha, \mathrm{n})$

\footnotetext{
3 Web page: http://www.lngs.infn.it
}

reactions and spontaneous fission of ${ }^{238} \mathrm{U}$ taking place in the surrounding rock and concrete [34], the measured total neutron flux is higher, $\Phi_{\mathrm{n}} \approx 4 \cdot 10^{-6} \frac{\mathrm{n}}{\mathrm{cm}^{2} \mathrm{~s}}$ [35]. This flux is three orders of magnitude below typical values for a laboratory at the surface of the earth.

This unique low background environment reduces the counting rate at $6.8 \mathrm{MeV}$ in a germanium detector by at least a factor 2000 , and at $6.5-8.0 \mathrm{MeV}$ in a BGO detector by a factor 1600 [36]. For comparison, an active muon shield in a laboratory at the surface of the earth can reduce the background counting rate by about a factor $10-50$ for $E_{\gamma}=7-11 \mathrm{MeV}$ [37]. The shield provided by the Gran Sasso rock cover therefore offers a clear advantage, in particular at high $\gamma$ energies, but also at low $\gamma$ energies and for particle spectroscopy.

Taking advantage of the low laboratory background, at the $50 \mathrm{kV}$ LUNA1 accelerator [38], the ${ }^{3} \mathrm{He}\left({ }^{3} \mathrm{He}, 2 \mathrm{p}\right){ }^{4} \mathrm{He}$ cross section was measured for the first time within its solar Gamow peak [39,40]. Subsequently, a windowless gas target setup and a $4 \pi$ bismuth germanate (BGO) summing detector [41] have been used to study the radiative capture reaction ${ }^{2} \mathrm{H}(\mathrm{p}, \gamma)^{3} \mathrm{He}$, also within its solar Gamow peak [42].

The $400 \mathrm{kV}$ LUNA2 accelerator [43] has been used to study the radiative capture reaction ${ }^{14} \mathrm{~N}(\mathrm{p}, \gamma){ }^{15} \mathrm{O}$ using titanium nitride $(\mathrm{TiN})$ solid targets and a high purity germanium detector. The cross sections for the transitions to several states in ${ }^{15} \mathrm{O}$, including the ground state, were measured down to $E=119 \mathrm{keV}[44,22,45]$.

In order to extend the ${ }^{14} \mathrm{~N}(\mathrm{p}, \gamma){ }^{15} \mathrm{O}$ cross section data to even lower energies, a gas target setup similar to the one used for the ${ }^{2} \mathrm{H}(\mathrm{p}, \gamma)^{3} \mathrm{He}$ study and an annular BGO detector have been installed at the LUNA2 $400 \mathrm{kV}$ accelerator [46].

\section{LUNA ${ }^{14} \mathrm{~N}(\mathrm{p}, \gamma){ }^{15} \mathrm{O}$ gas target experiment}

A new measurement of the total cross section of the ${ }^{14} \mathrm{~N}(\mathrm{p}, \gamma){ }^{15} \mathrm{O}$ reaction $[6,7]$ has been performed in the Gran Sasso underground laboratory, at the LUNA2 $400 \mathrm{kV}$ accelerator [43]. The main features of the experiment are described in this section.

\subsection{Setup}

A schematic view of the setup is displayed in fig. 6. A three stage, differentially pumped, windowless gas target system (figs. 6 and 7) has been used. It is a modified version of the LUNA ${ }^{2} \mathrm{H}(\mathrm{p}, \gamma)^{3} \mathrm{He}$ setup [41], with a $120 \mathrm{~mm}$ long target cell. In the experiment, a proton beam of energy $E_{\mathrm{p}}=80-250 \mathrm{keV}$ and current up to $0.5 \mathrm{~mA}$ is provided by the LUNA2 $400 \mathrm{kV}$ accelerator and enters the target chamber through a sequence of long, narrow, water cooled apertures; the final aperture has a diameter of $7 \mathrm{~mm}$, is $40 \mathrm{~mm}$ long and made from brass, with a copper cover on the side facing the ion beam. The target cell is fitted into the $60 \mathrm{~mm}$ wide bore hole at the center of an annular BGO detector having $70 \mathrm{~mm}$ radial thickness 


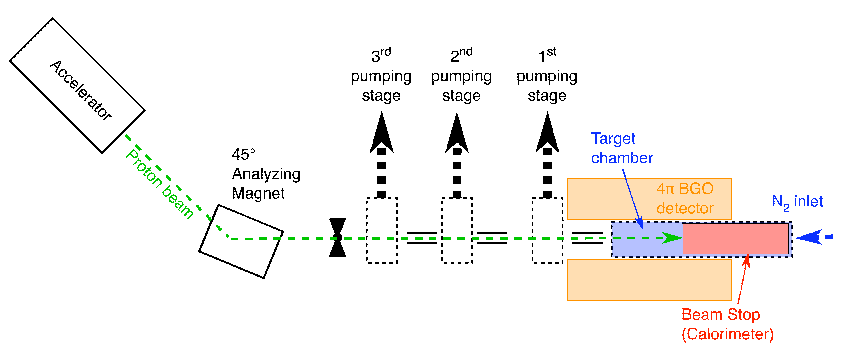

Fig. 6. Schematic view of the LUNA ${ }^{14} \mathrm{~N}(\mathrm{p}, \gamma){ }^{15} \mathrm{O}$ gas target setup.

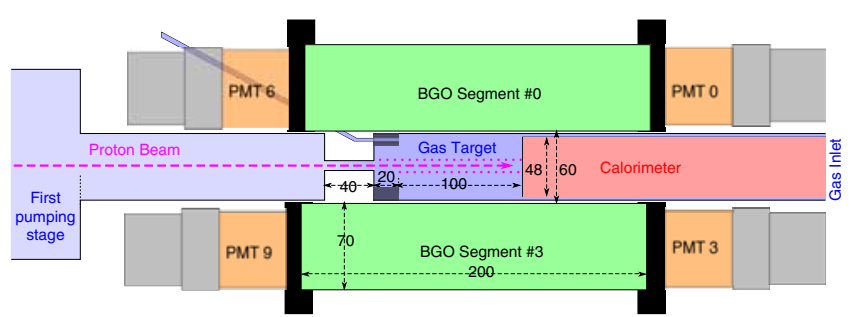

Fig. 7. Exploded view of the target chamber and $4 \pi$ BGO detector. Dimensions are given in $\mathrm{mm}$.

and $280 \mathrm{~mm}$ length. Also inside the BGO bore hole is a calorimeter (heated to $70^{\circ} \mathrm{C}$ ) for the measurement of the beam intensity, with a $41 \mathrm{~mm}$ thick block of oxygen free copper serving as the beam stop.

The target gas was 1.0 mbar nitrogen of chemical purity $99.9995 \%$ and natural isotopic composition, with 1.0 mbar helium gas of chemical purity $99.9999 \%$ used for monitor runs for ion beam induced background from the ${ }^{13} \mathrm{C}(\mathrm{p}, \gamma){ }^{14} \mathrm{~N}$ reaction $[36,6]$. The first pumping stage is evacuated by a WS 2000 roots blower, leading to a pressure ratio between target and first pumping stage that is better than a factor 100 . The second and third pumping stages are at $10^{-5}$ and $10^{-6}$ mbar pressure, respectively.

\subsection{Target density}

The target density without and with ion beam has been investigated in a dedicated study $[47,6,7]$. The target pressure was monitored with a capacitance pressure gauge with precision $0.1 \%$ and kept constant with a feedback system. The pressure profile within the target has been measured with similar precision and is flat to $4 \%$. The temperature profile without incident ion beam has been measured to better than $1 \mathrm{~K}$. To study the target density with an ion beam incident on the target, a collimated NaI detector was placed at an angle of $90^{\circ}$ to the beam direction directly next to the target chamber, and the energy loss $\Delta E_{\text {exp }}$ of the ion beam inside the target chamber was measured with the resonance scan technique [48] using the $E_{\mathrm{p}}=278 \mathrm{keV}$ resonance in the ${ }^{14} \mathrm{~N}(\mathrm{p}, \gamma){ }^{15} \mathrm{O}$ reaction. The experiment was repeated for different pressures and beam currents $I_{\text {target }}$ (fig. 8).

For high target pressure, therefore high power deposition per unit length in the target, there is a large relative effect on $\Delta E_{\text {exp }}$. As is evident from fig. 8, the relative

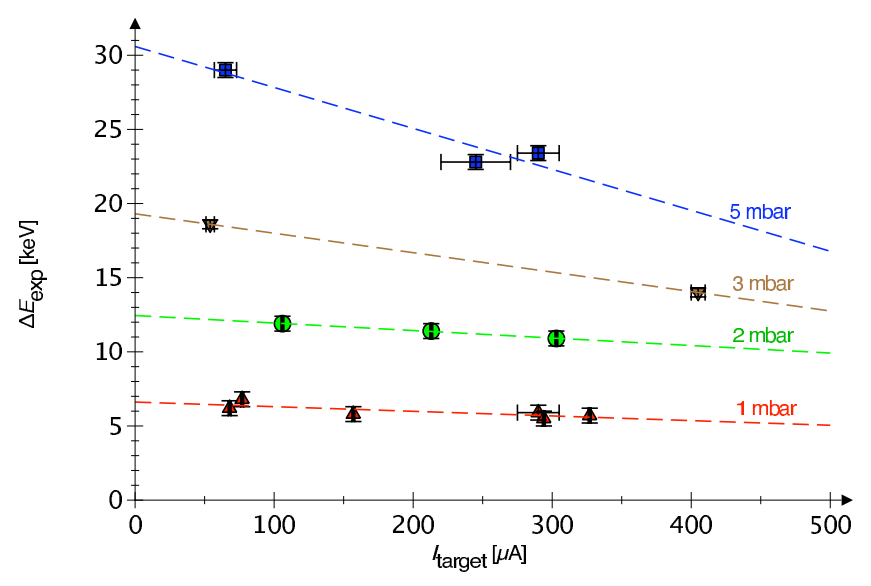

Fig. 8. Measured energy loss $\Delta E_{\exp }$ in the nitrogen target gas as a function of $I_{\text {target }}$ for different gas pressures. Triangles: 1 mbar; circles: 2 mbar; inverted triangles: 3 mbar; squares: 5 mbar.

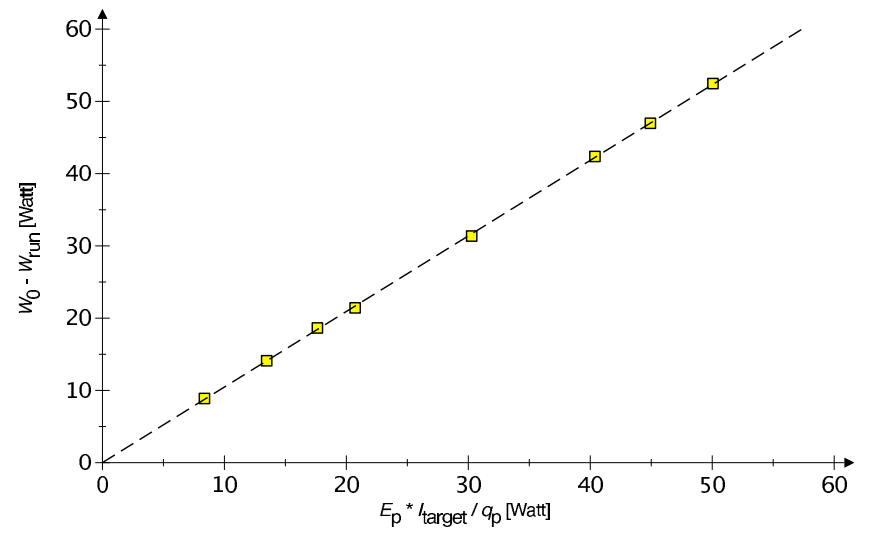

Fig. 9. Calorimetric power $W_{0}-W_{\text {run }}$ as a function of electrical power $\frac{E_{\mathrm{p}} \cdot I_{\text {target }}}{q_{\mathrm{p}}}$, with $q_{\mathrm{p}}$ the charge of the proton. The dotted line is a fit to the data points.

change in $\Delta E_{\exp }$ is also proportional to the beam current. Comparing $\Delta E_{\text {exp }}$ to the energy loss taken from the SRIM program [49], one obtains the particle density per unit volume. Consistent with the conclusions of ref. [48], the relative change in density was found to be proportional to the power deposited per unit length, which in the present case of small lateral straggling of the ion beam corresponds to the power deposited per unit volume.

\subsection{Beam intensity}

The intensity of the ion beam was measured with a calorimeter with constant temperature gradient [41]. The $41 \mathrm{~mm}$ thick copper beam stop forms the hot side of the calorimeter, that was kept at $70^{\circ} \mathrm{C}$ with thermoresistors (power consumption typically $135 \mathrm{~W}$ ). For the calibration of the calorimeter (fig. 9), the target chamber was used as a Faraday cup, a negative voltage was applied to the final collimator in order to repel secondary electrons, and the electrical target current $I_{\text {target }}$ was measured with a standard current integrator. Electrical and calorimetric cur- 


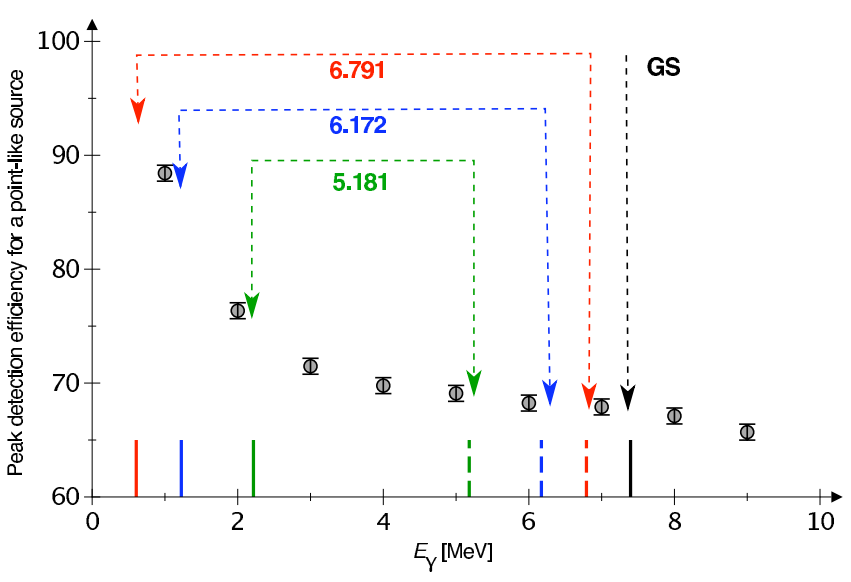

Fig. 10. Peak detection efficiency as a function of $\gamma$-ray energy [41]. The energies of the most important primary and secondary $\gamma$ lines are indicated (primary: solid line, secondary: dashed line), for center of mass energy $E=100 \mathrm{keV}$.

rent were found to agree with a slope $5 \%$ different from unity, and no offset within errors.

\subsection{Detection efficiency}

The peak detection efficiency of the $4 \pi$ BGO summing crystal as a function of $\gamma$-ray energy for a point-like source (fig. 10) has been given elsewhere [41].

For the analysis, all $\gamma$-rays detected in a region of interest (ROI) from 6 to $8 \mathrm{MeV}$ (figs. 11, 12) are summed. Therefore, the peak from true coincidence summing of a primary and its associated secondary $\gamma$-ray is fully within the ROI, as well as the primary $\gamma$-ray at $E_{\gamma}=$ $Q+E$ for capture into the ground state in ${ }^{15} \mathrm{O}(Q$ value $Q=7.297 \mathrm{MeV}$ for $\left.{ }^{14} \mathrm{~N}(\mathrm{p}, \gamma){ }^{15} \mathrm{O}\right)$, the secondary $\gamma$-ray at $6.791 \mathrm{MeV}$ and $80 \%$ of the peak area of the $6.172 \mathrm{MeV}$ secondary $\gamma$-ray. This selection of the ROI renders the detection efficiency independent of the branching ratios for capture to the ground state and to the state at $6.791 \mathrm{MeV}$, and only weakly dependent on the branching ratio for capture to the state at $6.172 \mathrm{MeV}$. The efficiency depends more strongly on the branching ratio for capture to the state at $5.181 \mathrm{MeV}$, but the impact is small because of the low value of the branching to this state: $3.6 \%$ branching at the lowest measured point [45], and $0.8 \%$ extrapolated at zero energy [20]. Overall, the assumptions on these four branching ratios contribute $0.5 \%$ to the uncertainty in the detection efficiency.

The $\gamma$-ray detection efficiency for radiative capture to the states at 7.276 and $6.859 \mathrm{MeV}$ in ${ }^{15} \mathrm{O}$ (fig. 4, extrapolated branching at zero energy 1 and $2 \%$, respectively [20]) is $20 \%$ lower than for capture to the state at $6.791 \mathrm{MeV}$, because those states decay to the ground state via the $5.241 \mathrm{MeV}$ state. The efficiency for capture into the $5.241 \mathrm{MeV}$ state (extrapolated branching at zero energy $1 \%[20]$ ) shows the same behavior. Capture into the three states at $7.276,6.859$ and $5.241 \mathrm{MeV}$ in ${ }^{15} \mathrm{O}$ has been neglected in the present experiment. If one assumes three times higher branching ratios at low energy for these

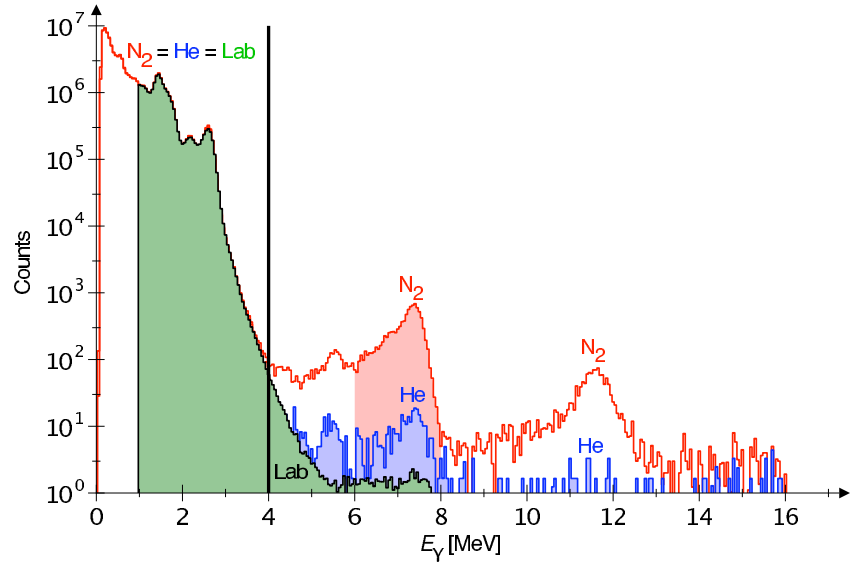

Fig. 11. $\mathrm{N}_{2}$ : Gamma-ray spectrum for $E_{\mathrm{p}}=140 \mathrm{keV}(E=$ $127 \mathrm{keV}$ ) with 1 mbar nitrogen gas, lifetime 47 hours, accumulated charge 45 coulomb. He: Same beam energy, 1 mbar helium in the target. For $E_{\gamma}<4 \mathrm{MeV}$, renormalized to equal lifetime with the $\mathrm{N}_{2}$ run. For $E_{\gamma}>4 \mathrm{MeV}$, renormalized to equal charge and proton energy at the beam stop. Lab: Laboratory background without beam, renormalized to equal lifetime with the $\mathrm{N}_{2}$ run.

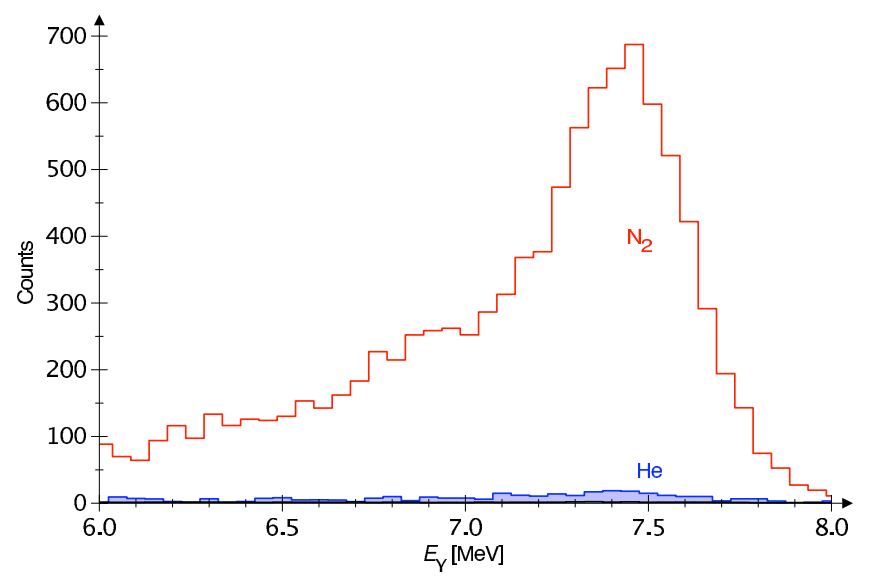

Fig. 12. Same spectrum as fig. 11, enlarged to the ROI. The Compton background to be subtracted for this spectrum corresponds to 5 counts per channel (not shown in the figure).

states than given by extrapolation [20], the total cross section obtained increases by $3 \%$.

In summary, while the calculated detection efficiency does depend on the branching ratios for capture into the different states as taken from the LUNA solid target experiment [45] and from R-matrix extrapolations for low energy $[22,24,20]$, this dependence is diluted by the particularities of the ${ }^{15} \mathrm{O}$ level scheme, the essentially flat peak detection efficiency curve for $5 \mathrm{MeV}<E_{\gamma}<8 \mathrm{MeV}$, and the choice of a wide ROI, so that the resultant systematic uncertainty is $1 \%$ for reasonable and $3 \%$ for worst case assumptions on the uncertainties of the branching ratios.

The angular distribution $W(\vartheta)$ of the emitted $\gamma$-rays has been studied previously in the LUNA solid target experiment [50], above and below the $E_{\mathrm{p}}=278 \mathrm{keV}$ reso- 


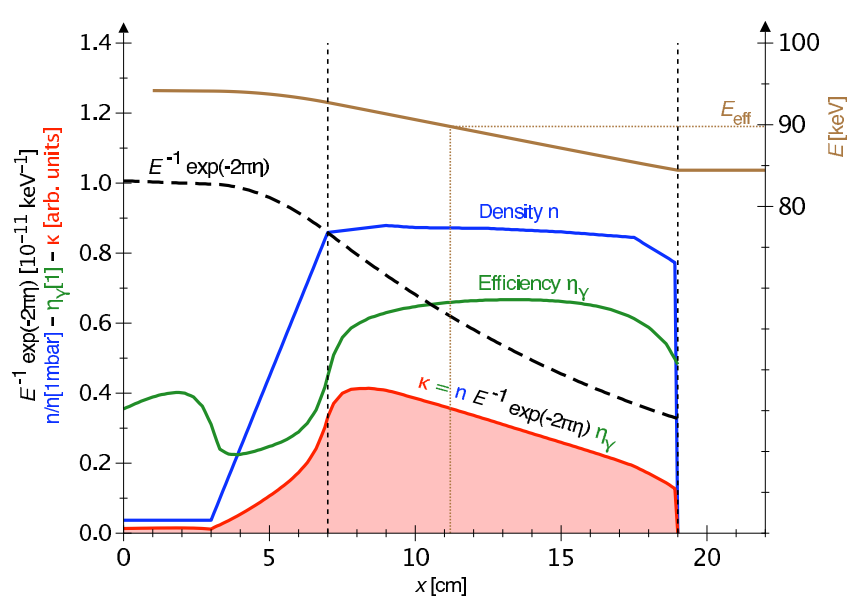

Fig. 13. Parameters used for the analysis in an example run at $E=90 \mathrm{keV}$. Right axis: energy $E(x)[\mathrm{keV}]$. Left axis: $E(x)^{-1} \exp (-2 \pi \eta)\left[10^{-11} \mathrm{keV}^{-1}\right]$. Effective target density $n(x) / n[1$ mbar, nobeam $]$; absolute $\gamma$-ray detection efficiency $\eta_{\gamma}$; weighting factor $\kappa$ for each piece of the target [arbitrary units].

nance; it can be parameterized as

$$
W(\vartheta) \approx 1+a_{1} \cdot P_{1}(\cos (\vartheta))+a_{2} \cdot P_{2}(\cos (\vartheta)),
$$

where $P_{1,2}$ are the first and second order Legendre coefficients and $\vartheta$ is the angle (in the center of mass system) between the ion beam and the direction of emission of the $\gamma$-ray.

All secondary $\gamma$-rays shown in fig. 10 were observed to be isotropic within errors, in agreement with theoretical expectation. For the primary $\gamma$-rays shown in fig. 10, theory predicts Legendre coefficients $a_{2}<0$ or $a_{2}=0$ for incident $s$ - and $p$-waves. The data show all primary $\gamma$-rays to be isotropic within errors, with the exception of that from capture to the state at $6.791 \mathrm{MeV}$, where $a_{2} \approx-0.8$ below the resonance. For all primary and secondary transitions, below the resonance the $a_{1}$ coefficient was found to be zero within errors [50].

An anisotropy with $a_{2}<0$ enhances emission perpendicular to the beam direction and therefore the detection efficiency for the low energy primary $\gamma$-ray, increasing the probability of it being detected in coincidence with the corresponding secondary $\gamma$-ray. For capture into the states at 6.791 and $6.172 \mathrm{MeV}$, the angular distribution of the primary $\gamma$-rays, while changing the shape of the spectrum, does not affect the detection efficiency, because the selection of the ROI ensures detection of both the secondary and the sum peak. For capture into the ground state (where theory predicts isotropy) and into the state at $5.182 \mathrm{MeV}$, there is an effect, but it is diluted because of the relatively small branching of those two states (combined less than 20\%). The overall impact of the angular distributions on the detection efficiency is smaller than $3 \%$ without theoretical input and negligible when taking theory into account.

Using these inputs, the $\gamma$-ray detection efficiency $\eta_{\gamma}$ can then be calculated for each point in the target, taking the solid angle and the effective detector thickness into account [6], with corrections for the attenuation of $\gamma$-rays in the vacuum vessel, the massive brass collimator and the massive copper beam stop. For the example shown in fig. 13, the detection efficiency is $\eta_{\gamma}=0.592 \pm 0.020$, with the uncertainty given by the radioactive source used for the calibration $(1.5 \%)$, the detector modeling (1\%, [41]) and the branching ratios discussed above (1\%).

\subsection{Gamma-ray spectra and background}

Using a dedicated setup, the $\gamma$-ray background has been studied previously to the actual experiment, identifying and localizing the major background sources $[36,6]$. Typical $\gamma$-ray spectra from the $4 \pi$ BGO summing detector are shown in fig. 11, with the region of interest (ROI) for the ${ }^{14} \mathrm{~N}(\mathrm{p}, \gamma){ }^{15} \mathrm{O}$ study shaded in the $\left(\mathrm{N}_{2}\right)$ spectrum.

For $E_{\gamma}<4 \mathrm{MeV}$, the spectrum is dominated by the laboratory background, whose counting rate in the ROI is constant and well known [36]. At higher $\gamma$-energies, the background induced by the ion beam is for most runs more important than the laboratory background. Background induced by the ${ }^{13} \mathrm{C}(\mathrm{p}, \gamma){ }^{14} \mathrm{~N}$ reaction $(Q=7.551 \mathrm{MeV})$ leads to $7.7 \mathrm{MeV} \gamma$-rays, superimposed with the sum peak from the reaction to be studied. In order to evaluate the contribution from this reaction, monitor runs with helium gas in the target were performed at the same beam energy. The resulting monitor spectrum is then renormalized for equal charge with the nitrogen spectrum and for equal energy of the proton beam when arriving at the beam stop, where the ${ }^{13} \mathrm{C}$ background originates ( $(\mathrm{He})$ spectrum in fig. 11).

In the nitrogen $\left(\mathrm{N}_{2}\right)$ spectrum, the dominating peak in the ROI (fig. 12) is the sum peak at $E_{\gamma}=Q+E$. To the left of it are unresolved lines at 6.172 and $6.791 \mathrm{MeV}$, the energies of the secondary $\gamma$-rays. Outside the ROI, the peak at $5 \mathrm{MeV}$ (fig. 11) is mostly from the secondary $\gamma$-ray at $5.181 \mathrm{MeV}$ from the reaction to be studied, but partly also from the ${ }^{2} \mathrm{H}(\mathrm{p}, \gamma){ }^{3} \mathrm{He}$ beam induced background reaction, as is revealed by the helium monitor run.

The broad structure at $12 \mathrm{MeV}$ in the $\mathrm{N}_{2}$ spectrum (fig. 11) results mainly from the ${ }^{15} \mathrm{~N}(\mathrm{p}, \gamma){ }^{16} \mathrm{O}$ reaction (the target gas has natural isotopic composition, $\left.0.4 \%{ }^{15} \mathrm{~N}\right)$, but also from the ${ }^{11} \mathrm{~B}(\mathrm{p}, \gamma){ }^{12} \mathrm{C}$ beam induced background reaction. This last reaction also gives $\gamma$-rays at $16 \mathrm{MeV}$. All reactions leading to $\gamma$-rays of $E_{\gamma}>8 \mathrm{MeV}$ [36] cause a small Compton continuum at lower energies. Its contribution is evaluated from a global fit to the helium monitor runs (after ${ }^{13} \mathrm{C}$ correction), and a correction factor is deduced, so that the high energy counts in each spectrum are used to calculate the Compton background for that same spectrum [6]. Finally, single lines from resonant background reactions producing $\gamma$-rays in the ROI [36] were fitted and subtracted for runs close to the resonance energy.

\subsection{Data analysis}

With the $\gamma$-ray detection efficiency $\eta_{\gamma}$, the effective target density $n$ and therefore also the energy loss of the ion 
beam in the target (in the present case, typically $10 \mathrm{keV}$ ) known, a weighting factor $\kappa(x)$ is calculated for each point $x$ in the target:

$$
\kappa(x) \stackrel{\text { Def }}{=} n \cdot \frac{1}{E(x)} e^{-2 \pi \eta} \cdot \eta_{\gamma}
$$

with $2 \pi \eta$ the Sommerfeld parameter from eq. (2). The parameter $\kappa(x)$ (fig. 13) can then be used to calculate the effective energy $E_{\text {eff }}$ and, using the measured yield $Y$, the astrophysical $S$-factor $S\left(E_{\text {eff }}\right)[1,6]$ :

$$
\begin{aligned}
Y & =\int_{x=0 \mathrm{~cm}}^{28 \mathrm{~cm}} \sigma(E(x)) \cdot n(x) \cdot \eta_{\gamma}(x) \mathrm{d} x= \\
& =S\left(E_{\mathrm{eff}}\right) \cdot \int_{x=0 \mathrm{~cm}}^{28 \mathrm{~cm}} \kappa(x) \mathrm{d} x .
\end{aligned}
$$

This analysis method requires an assumption on the energy dependence of the $S$-factor. In the present experiment, as a first step the analysis has been performed under the assumption of an $S$-factor that is constant over the energy interval given by the energy loss in the target. In a second step, the obtained energy dependence of the $S$-factor has been used as input for the renewed analysis.

Using this method, total cross section data with statistical uncertainties better than $10 \%$ has been obtained in the energy range $E=70-230 \mathrm{keV}$, energies lower than any previous study.

\section{Astrophysical scenarios that can be better understood using data from the present experiment}

The data obtained in the present experiment $[6,7]$ can be used to directly evaluate the reaction rate for several important stellar scenarios, with negligible impact from the extrapolation applied for lower energies.

The derivative $\frac{\mathrm{d}\langle\sigma v\rangle}{\mathrm{d} E}$ of the reaction rate from eq. (1) has been calculated from the LUNA gas target experimental $S$-factor data [6], assuming a flat $S$-factor equal to the $S$-factor at $E=70 \mathrm{keV}$ for $E<70 \mathrm{keV}$, where there is no data (fig. 14). For temperatures $T_{6} \geq 60$, the data from the present experiment cover more than $50 \%$ of the Gamow peak, for $90 \leq T_{6} \leq 300$, more than $90 \%$ of the Gamow peak, when one includes the strength of the $E=259 \mathrm{keV}$ resonance that was also measured in the LUNA gas target experiment [7].

Low mass stars burn first hydrogen and then helium in their center. After the end of the helium burning phase, the star consists of a degenerate core of oxygen and carbon and two shells burning hydrogen and helium, respectively. This phase of stellar evolution is called the asymptotic giant branch (AGB) [51]. It is characterized by flashes of the helium burning shell that spawn convective mixing in a process called dredge-up. Such a dredge-up transports the products of nuclear burning from inner regions of the

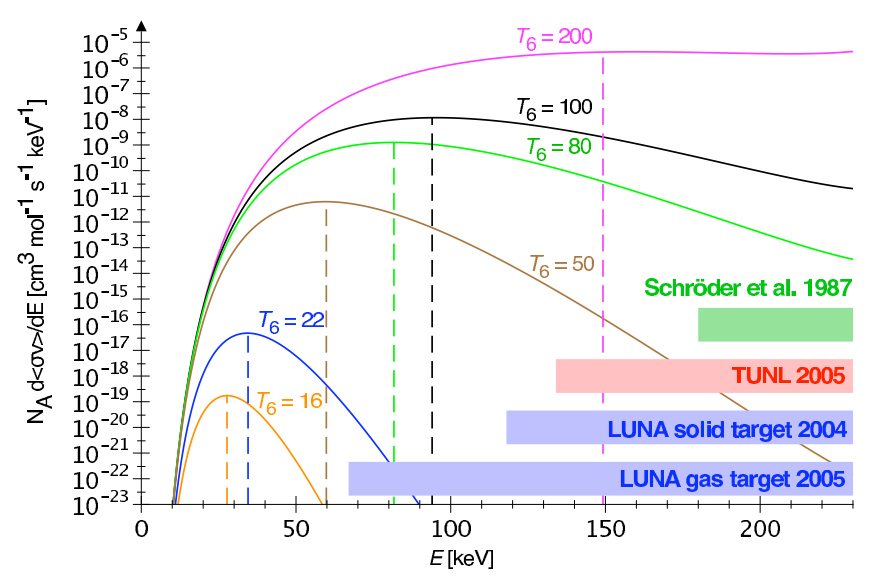

Fig. 14. Gamow peaks for several stellar temperatures discussed in the text. The horizontal bars correspond to the energy range where direct experimental data has been obtained in the study by Schröder et al. 1987 [20], the LUNA solid target experiment 2004 [22], the TUNL 2005 study [29], and the LUNA gas target experiment (present work).

star to its surface, where they are in principle accessible to astronomical observations.

The temperature in the hydrogen burning shell of an AGB star is of the order of $T_{6}=50-80$ for the example of a $2 M_{\odot}$ star with metallicity $Z=0.01$. It has been shown [9] that an arbitrary $25 \%$ reduction of the ${ }^{14} \mathrm{~N}(\mathrm{p}, \gamma){ }^{15} \mathrm{O}$ rate with respect to the NACRE [4] rate leads to twice as efficient dredge-up of carbon to the surface of the star, because the rate of energy generation in the hydrogen burning shell becomes even lower than before, enhancing the disequilibrium between hydrogen and helium burning shell. The CNO rate suggested by the present study $[6,7]$ is more than $25 \%$ below the NACRE [4] rate. Recent experimental data on the carbon producing triple- $\alpha$ reaction [52] result in a 10-20\% decrease of its rate at temperatures relevant for helium shell burning, leading to a slightly lower production of carbon, reducing in a commensurate decrease of the amount of carbon transported to the stellar surface [9]. Still, the change in the ${ }^{14} \mathrm{~N}(\mathrm{p}, \gamma){ }^{15} \mathrm{O}$ rate might lift a disagreement between model and observation for so-called carbon stars [53]: For low (i.e. $2 M_{\odot}$ ) mass stars, models do not reproduce a sufficiently high dredgeup efficiency.

Recently, a simulation for a $5 M_{\odot}, Z=0.02$ AGB star [54] found stronger thermal flashes for a reduced CNO rate, consistent with the finding of ref. [9] for a $2 M_{\odot}$, $Z=0.01$ AGB star.

For a zero metallicity (population III) star of $1 M_{\odot}$, after a sufficient amount of carbon has been created in the triple- $\alpha$ reaction, the $\mathrm{CNO}$ cycle is ignited in the so called $\mathrm{CN}$ flash. This $\mathrm{CN}$ flash takes place at $T_{6} \approx 65$ and leads to a brief loop of the trajectory of the star in the Hertzsprung-Russell diagram [55]. With a CNO rate that is $40 \%$ lower than the NACRE [4] rate, this loop disappears [54]. Also, the first core helium flash in such a star was found to be less luminous than in the reference 
case, albeit with a higher core mass, as a result of a lower $\mathrm{CNO}$ rate [54].

Temperatures of $T_{6} \approx 100$ correspond to CNO burning in heavy $\left(20 M_{\odot}\right)$ population III stars [8]. Explosive burning in novae [10] takes place at even higher temperatures, typically $T_{6} \approx 200$. The $\frac{15 \mathrm{~N}}{14 \mathrm{~N}}$ isotopic ratio in nova ashes depends sensitively on the ${ }^{14} \mathrm{~N}(\mathrm{p}, \gamma){ }^{15} \mathrm{O}$ rate [56]; the more precise rate that can be calculated from the cross sections obtained in the present study will reduce the uncertainty of the isotopic ratio.

In conclusion, data from the present study allow for the first time to directly evaluate the reaction rate for several scenarios of stable stellar hydrogen burning, as well as for explosive hydrogen burning.

During the experiment, D. Bemmerer was Wissenschaftlicher Mitarbeiter at the Institut für Atomare Physik und Fachdidaktik, Technische Universität Berlin, Germany. This work was supported in part by: INFN, TARI HPRI-CT-200100149, OTKA T 42733, BMBF (05CL1PC1-1), FEDERPOCTI/FNU/41097/2001, and EU RII3-CT-2004-506222.

\section{References}

1. C. Rolfs, W. Rodney, Cauldrons in the Cosmos (University of Chicago Press, Chicago, 1988).

2. H. Bethe, Phys. Rev. 55, 434 (1938).

3. J.N. Bahcall, M.H. Pinsonneault, Phys. Rev. Lett. 92, 121301 (2004).

4. C. Angulo et al., Nucl. Phys. A 656, 3 (1999).

5. M. Wiescher, J. Görres, H. Schatz, J. Phys. G 25, R133 (1999).

6. D. Bemmerer, Experimental study of the ${ }^{14} \mathrm{~N}(\mathrm{p}, \gamma)^{15} \mathrm{O}$ reaction at energies far below the Coulomb barrier, $\mathrm{PhD}$ Thesis, Technische Universität Berlin (2004).

7. A. Lemut, Misura della sezione d'urto della reazione ${ }^{14} \mathrm{~N}(\mathrm{p}, \gamma){ }^{15} \mathrm{O}$ ad energie di interesse astrofisico, $\mathrm{PhD}$ Thesis, Università degli Studi di Genova (2005).

8. L. Siess, M. Livio, J. Lattanzio, Astrophys. J. 570, 329 (2002).

9. F. Herwig, S. M. Austin, Astrophys. J. 613, L73 (2004).

10. J. José, M. Hernanz, Astrophys. J. 494, 680 (1998).

11. J.N. Bahcall, A.M. Serenelli, S. Basu, Astrophys. J. 621, L85 (2005).

12. L. Krauss, B. Chaboyer, Science 299, 65 (2003).

13. G. Imbriani et al., Astron. Astrophys. 420, 625 (2004).

14. E. Degl'Innocenti et al., Phys. Lett. B 590, 13 (2004).

15. D.B. Duncan, J.E. Perry, Phys. Rev. 82, 809 (1951).

16. W. Lamb, R. Hester, Phys. Rev. 108, 1304 (1957).

17. R.E. Pixley, The reaction cross section of nitrogen 14 for protons between $220 \mathrm{keV}$ and $600 \mathrm{keV}, \mathrm{PhD}$ Thesis, California Institute of Technology (1957).

18. B. Povh, D.F. Hebbard, Phys. Rev. 115, 608 (1959).
19. D.F. Hebbard, G.M. Bailey, Nucl. Phys. 49, 666 (1963).

20. U. Schröder et al., Nucl. Phys. A 467, 240 (1987).

21. F. Ajzenberg-Selove, Nucl. Phys. A 523, 1 (1991).

22. A. Formicola et al., Phys. Lett. B 591, 61 (2004).

23. E. Adelberger et al., Rev. Mod. Phys. 70, 1265 (1998).

24. C. Angulo, P. Descouvemont, Nucl. Phys. A 690, 755 (2001).

25. P. Bertone et al., Phys. Rev. Lett. 87, 152501 (2003).

26. P.F. Bertone et al., Phys. Rev. C 66, 055804 (2002).

27. A. Mukhamedzhanov et al., Phys. Rev. C 67, 065804 (2003).

28. K. Yamada et al., Phys. Lett. B 579, 265 (2004).

29. R.C. Runkle et al., Phys. Rev. Lett. 94, 082503 (2005).

30. A.M. Mukhamedzhanov, C.A. Gagliardi, R.E. Tribble, Phys. Rev. C 63, 024612 (2001).

31. S.O. Nelson et al., Phys. Rev. C 68, 065804 (2003).

32. S.P. Ahlen et al., Phys. Lett. B 249, 149 (1990).

33. H. Wulandari et al., (2004), hep-ex/0401032.

34. H. Wulandari, J. Jochum, W. Rau, F. von Feilitzsch, Astropart. Phys. 22, 313 (2004).

35. P. Belli et al., Nuovo Cimento A 101, 959 (1989).

36. D. Bemmerer et al., Eur. Phys. J. A 24, 313 (2005).

37. G. Müller et al., Nucl. Instrum. Methods A 295, 133 (1990).

38. U. Greife et al., Nucl. Instrum. Methods A 350, 327 (1994).

39. M. Junker et al., Phys. Rev. C 57, 2700 (1998).

40. R. Bonetti et al., Phys. Rev. Lett. 82, 5205 (1999).

41. C. Casella et al., Nucl. Instrum. Methods A 489, 160 (2002).

42. C. Casella et al., Nucl. Phys. A 706, 203 (2002).

43. A. Formicola et al., Nucl. Instrum. Methods A 507, 609 (2003).

44. A. Formicola et al., Nucl. Phys. A 719, 94c (2003).

45. A. Formicola, A new study of ${ }^{14} \mathrm{~N}(\mathrm{p}, \gamma)^{15} \mathrm{O}$ at low energy, PhD Thesis, Ruhr-Universität Bochum (2004).

46. LUNA Collaboration, LNGS Annual Report, 159 (2003).

47. F. Confortola, Studio della reazione ${ }^{14} \mathrm{~N}(\mathrm{p}, \gamma){ }^{15} \mathrm{O}$ ad energie di interesse astrofisico, Master's Thesis, Università degli Studi di Genova (2003).

48. J. Görres et al., Nucl. Instrum. Methods 177, 295 (1980).

49. J. Ziegler, SRIM version 2003.26, http://www.srim.org (2004).

50. H. Costantini, Direct measurements of radiative capture reactions at astrophysical energies, $\mathrm{PhD}$ Thesis, Università degli Studi di Genova (2003).

51. I. Iben, A. Renzini, Annu. Rev. Astron. Astrophys. 21, 271 (1983).

52. H. Fynbo et al., Nature 433, 136 (2005).

53. I. Iben, Astrophys. J. 246, 278 (1981).

54. A. Weiss, A. Serenelli, A. Kitsikis, H. Schlattl, J. Christensen-Dalsgaard, astro-ph/0503408 (2005).

55. A. Weiss, S. Cassisi, H. Schlattl, M. Salaris, Astrophys. J. 533, 413 (2000).

56. C. Iliadis, A. Champagne, J. José, S. Starrfield, P. Tupper, Astrophys. J. Suppl. Ser. 142, 105 (2002). 